\title{
Risk factors for the occurrence and persistence of coronary aneurysms in Kawasaki disease
}

\author{
Soo-Kyeong Jeon, MD, Geena Kim, MD, Hoon Ko, MD, Joung-Hee Byun, MD, Hyoung Doo Lee, MD, PhD \\ Department of Pediatrics, Pusan National University Children's Hospital, Yangsan, Korea
}

Purpose: Prognostic factors of coronary aneurysms in Kawasaki disease have been investigated in many studies. The aim of this study was to identify risk factors associated with early and late coronary artery outcomes in treated patients with Kawasaki disease.

Methods: A total of 392 patients diagnosed with Kawasaki disease from January 2012 to December 2015 in Pusan National University Children's Hospital were retrospectively selected as subjects of the present study to determine risk factors for coronary aneurysms and persistence of coronary aneurysms after a 1-year follow-up.

Results: Coronary aneurysms were detected in 30 of 392 patients within 1 month after the occurrence of Kawasaki disease. Coronary aneurysms persisted in 5 of 30 patients after a 1-year follow-up. A long duration of fever (adjusted odds ratio [OR], 1.47; 95\% confidence interval [Cl], 1.06-2.02; $P=0.018$ ) and high platelet count (adjusted OR, 1.00; $95 \% \mathrm{Cl}, 1.00-1.01 ; P=0.009$ ) were found to be independent factors to predict the development of coronary aneurysms in the early phase. Initial coronary severity (adjusted OR, 46.0; 95\% Cl, 2.01-1047.80; $P=0.016$ ) and a high white blood cell count (adjusted OR, $1.17 ; 95 \% \mathrm{Cl}, 1.01-1.36 ; P=0.028$ ) were found to be significant factors for the persistence of late coronary aneurysms in univariate analysis. However, no significant factors were found in multivariate analysis. Conclusion: These data are from early and late follow-up of coronary aneurysms in our unit. Further studies are needed to determine the mechanisms involved in the disappearance of coronary aneurysms and related factors.

Key words: Kawasaki disease, Coronary aneurysm, Prognosis

\section{Introduction}

Kawasaki disease is an acute febrile disease characteristically found in very young children or infants aged less than 6 years. ${ }^{1,2)}$ This disease was reported by Tomisaki Kawasaki in 1967..$^{1,3-}$

${ }^{5)}$ Its diagnostic criteria include a persistence of a fever of over $38^{\circ} \mathrm{C}$ for over 5 days while its clinical symptoms include nonsuppurative bilateral conjunctival injection, red lips, strawberry tongue, atypical exanthema, cervical lymph node enlargement, swelling and erythema of hands and feet, and membranous desquamation. ${ }^{1,3,67)}$ Most cases of Kawasaki disease are tractable by corresponding treatments without causing complications. However, this disease may accompany diverse cardiovascular complications such as coronary artery enlargement, coronary aneurysm, heart failure, and myocardial infarction. ${ }^{2,8}$

Previous studies have identified risk factors for patients showing coronary artery disorders among children suffering Kawasaki disease, including age less than one year, male sex, longlasting febrile stage over 14 days, higher level of total white blood cell (WBC) count, lower level of hemoglobin count, higher level of platelet count, higher level of erythrocyte sedimentation rate, higher level of C-reactive protein (CRP), and lower level of albumin. ${ }^{3,9)}$ Papers on long-
Corresponding author: Geena Kim, MD

Department of Pediatrics, Pusan National University Children's Hospital, 20 Geumo-ro, Mulgeumeup, Yangsan 50612, Korea

Tel: $+82-55-360-3600$

Fax: +82-55-360-2181

E-mail:drgnkim@naver.com

https://orcid.org/0000-0002-6837-7475

Received: 11 September, 2018

Revised: 1 November, 2018

Accepted: 22 November, 2018
Copyright $@ 2019$ by The Korean Pediatric Society

This is an open-access article distributed under the terms of the Creative Commons Attribution NonCommercial License (http://creativecommons.org/ licenses/by-nc/4.0/) which permits unrestricted noncommercial use, distribution, and reproduction in any medium, provided the original work is properly cited. 
term prognosis of Kawasaki disease have showed diversified analyses according to respective examinations and researchers. ${ }^{8)}$ The objective of the present study was to identify risk factors for the occurrence and persistence of coronary aneurysms in Kawasaki disease using patients who manifested acute phase coronary aneurysms with progress of coronary aneurysms for more than a year.

\section{Materials and methods}

\section{Patients}

Subjects of the present study were selected from 419 children who took in-patient care for Kawasaki disease in the Children's Hospital of Pusan National University from January 2012 to December 2015. These patients satisfied the diagnostic criteria for Kawasaki disease established in 2004 by the American Heart Association (AHA). ${ }^{10)}$ Medical records of a total of 392 subjects were analyzed retrospectively. Two children who had insufficient records and 25 children who did not take in-patient care were excluded. The age, sex, height, weight, and clinical symptoms of the children at the time of diagnosis were examined, as well as the presence of complete Kawasaki disease, findings from blood examinations, treatment methods, Harada scores, results of echocardiographies, and recurrences of the disease. ${ }^{11)}$ To determine the number of cases with complete Kawasaki disease, diagnostic criteria such as duration of the febrile stage of over 5 days and four clinical symptoms among five clinical symptoms established in 2004 by AHA were taken into account while diagnostic criteria specified for treatment of incomplete Kawasaki disease were used to determine the case. ${ }^{10)}$

\section{Echocardiography}

Patients underwent echocardiographies at 2 weeks, 2 months, and 1 year after admission for in-patient treatment. Determination of acute phase coronary artery disorder was based on the findings of echocardiographies conducted at 2 weeks after admission. ${ }^{12,13)}$ Cases with a coronary artery inner diameter of less than $5 \mathrm{~mm}$ were classified as small aneurysms whereas cases with a coronary artery inner diameter of 5-8 $\mathrm{mm}$ and over $8 \mathrm{~mm}$ were classified as medium aneurysms and large aneurysms, respectively ${ }^{14}$ If a coronary artery is larger than normal (dilated) without a segmental aneurysm, the vessel is ectatic. ${ }^{10)}$ The subjects were divided into 2 groups based on the presence or absence of coronary aneurysms from the point of diagnosis of Kawasaki disease.

\section{Treatment of Kawasaki disease}

Treatment of Kawasaki disease was initiated with intravenous immunoglobulin (IVIG, $2 \mathrm{~g} / \mathrm{kg}$ as single infusion) and a high dose of (50 mg/kg/day). Immunoglobulin was administered again if fever persisted for at least 36 hours after the end of their IVIG infusion. ${ }^{1,10)}$ Methyl prednisolone pulse $(30 \mathrm{mg} / \mathrm{kg}$ ) and infliximab were administered sequentially when there was no response to readministration of immunoglobulin. The dose of aspirin was reduced to $3-5 \mathrm{mg} / \mathrm{kg} /$ day three days after the initial administration. Such reduced administration was then continued for 8 weeks in cases where no coronary aneurysm was found. Otherwise, clopidogrel and warfarin were administered depending on the size of the coronary aneurysm to normalize symptoms.

Risk factors of acute phase coronary aneurysm were analyzed. Patients were followed up with for one year to conduct additional analysis of risk factors after checking for recovery from the disease.

\section{Statistics}

IBM SPSS Statistics ver. 21.0 (IBM Co., Armonk, NY, USA) was used for all statistical analyses. Statistical significance was considered at $P<0.05$. Data were presented as mean \pm standard deviation, number $(\%)$, or median (interquartile range), as appropriate. Variables were compared between 2 groups using the unpaired Student's $t$ test, the Mann-Whitney test, the Pearson chi-square test, and 1-way analysis of variance. The odds ratio (OR) with 95\% confidence interval (CI) was calculated using a logistic regression analysis to determine risk factors for early and late coronary aneurysm. This study was approved by the Institutional Review Board (IRB) of Pusan National University Yangsan Hospital (approval number: 05-2018-154). Written informed consent was waived by the IRB.

\section{Results}

Of 392 children who took in-patient care for the treatment of Kawasaki disease, 245 (62\%) were males and 147 (38\%) were females. The average age, height, and weight of these subjects at the time of diagnosis of Kawasaki disease were $30.6 \pm 20.9$ months, $90.57 \pm 15.0 \mathrm{~cm}$, and $14.1 \pm 11.5 \mathrm{~kg}$, respectively. Regarding clinical symptoms of Kawasaki disease, conjunctival injection in 364 subjects (92.8\%), cervical lymph node enlargement in 218 subjects (55.6\%), red lips in 337 subjects (85.9\%), atypical exanthema in 327 subjects (83.4\%), and swelling and erythema of hands and feet in 241 subjects (61.4\%) were identified. Echocardiography was performed on children at 2 weeks after initiation of in-patient care for Kawasaki disease. Results showed that 30 children (7\%) manifested coronary aneurysms whereas 362 children (93\%) had no coronary aneurysms. All subjects selected for the present study were then divided into 2 groups: group 1, comprising those who exhibited no lesions of acute phase coronary aneurysms; group 2, comparing those who manifested lesions of acute phase coronary aneurysms (Table 1). Group 2 showed significantly shorter duration of the febrile stage than group 1 (5.3 \pm 1.4 days vs. $7.2 \pm 2.4$ days, $P<0.001$ ). There was no significant difference in the presence of incomplete Kawasaki disease or other clinical symptoms between the 2 groups. Recurrence rate of Kawasaki disease in group 2 was significantly 
higher than that in group 1 (23.3\% vs. 6.3\%, $P=0.001$ ).

Results of WBC, hemoglobin, platelet, absolute neutrophil count, aspartate aminotransferase, alanine aminotransferase, protein, albumin, bilirubin, sodium, and CRP were reviewed. They are summarized in Table 2. When amounts of hemoglobin and albumin were compared between the 2 groups, group 2 had significantly lower levels of hemoglobin and albumin than group 1. In terms of platelet count and CRP level, group 1 showed significantly lower

Table 1. Demographic and clinical characteristics of patients

\begin{tabular}{lcccc}
\hline Characteristic & $\begin{array}{c}\text { Group 1 } \\
(\mathrm{n}=362)\end{array}$ & $\begin{array}{c}\text { Group 2 } \\
(\mathrm{n}=30)\end{array}$ & $\begin{array}{c}\text { Total } \\
(\mathrm{n}=392)\end{array}$ & $P$ value \\
\hline Sex, male:female & $222: 140$ & $23: 7$ & $245: 147$ & 0.131 \\
Age at diagnosis (mo) & $30.2 \pm 20.4$ & $36.5 \pm 26.2$ & $30.6 \pm 20.9$ & 0.117 \\
Height (cm) & $90.2 \pm 14.7$ & $95.8 \pm 19.7$ & $90.6 \pm 15.0$ & 0.073 \\
Weight (kg) & $13.5 \pm 4.4$ & $23.3 \pm 39.5$ & $14.2 \pm 11.6$ & 0.200 \\
Complete KD & $240(66.2)$ & $21(70.0)$ & $262(66.8)$ & 0.514 \\
Symptoms and signs & & & & \\
Days of fever & $5.3 \pm 1.4$ & $7.2 \pm 2.4$ & $5.5 \pm 1.6$ & $<0.001$ \\
Conjunctival injection & $335(91.7)$ & $29(96.6)$ & $364(92.8)$ & 0.442 \\
Cervical lymphadenopathy & $197(64.5)$ & $21(70.0)$ & $218(55.6)$ & 0.586 \\
Changes in lips and oral cavity & $312(86.5)$ & $25(83.3)$ & $337(85.9)$ & 0.145 \\
Polymorphous exanthema & $302(83.4)$ & $25(83.3)$ & $327(83.4)$ & 0.931 \\
Change in extremities & $219(59.2)$ & $22(73.3)$ & $241(61.5)$ & 0.106 \\
Recurrence & $24(6.3)$ & $7(23.3)$ & $31(7.5)$ & 0.001 \\
$\quad$ 1st & $12(3.3)$ & $3(10.0)$ & $15(3.8)$ & \\
2nd & $5(1.4)$ & $1(3.3)$ & $6(1.5)$ & \\
$>$ 3rd & $3(0.8)$ & $2(6.6)$ & $5(1.2)$ &
\end{tabular}

Values are presented as mean \pm standard deviation or numbers (\%).

Group 1, those who exhibited no lesions from acute phase coronary aneurysms; group 2, those who manifested lesions from acute phase coronary aneurysms; KD, Kawasaki disease. platelet counts and CRP level than group 2. In this study, comparing the 2 groups by their Harada scores, it was found that the mean score of group 1 was $3.6 \pm 1.1$ points, the mean score of group 2 was $4.0 \pm 1.2$ points, and group 2 had higher Harada scores than group 1 $(P=0.115)$.

Of 392 subjects, 40 subjects (10.2\%) showed treatment failure after the first administration of IVIG. Therefore, they took a second treatment of IVIG. Ten subjects (2.5\%) were administered with methyl prednisolone while infliximab was administered to only 1 subject (0.2\%). Group 2 had a relatively high number of administrations of IVIG (twice) with administration of methyl prednisolone for 7 cases (23.3\%, $P=0.02$ ), compared to 4 cases for group 1 (13.3\%, $P=0.004)$. In group 2 , only 5 subjects were found with coronary aneurysms based on echocardiographies conducted 1 year later (Table 3).

Thirty subjects manifested lesions of acute phase coronary aneurysm whereas 24 subjects exhibited small aneurysm. The rest of the subjects manifested medium aneurysms (3 subjects) and large

\begin{tabular}{lrrrr}
\multicolumn{5}{l}{ Table 3. Treatment and prognosis of patients } \\
\hline Variable & $\begin{array}{c}\text { Group 1 } \\
(\mathrm{n}=362)\end{array}$ & $\begin{array}{c}\text { Group 2 } \\
(\mathrm{n}=30)\end{array}$ & $\begin{array}{c}\text { Total } \\
(\mathrm{n}=392)\end{array}$ & Pvalue \\
\hline Treatment & 329 & 23 & 352 & 0.000 \\
1st Immunoglobulin & 27 & 3 & 30 & \\
2nd Immunoglobulin & 6 & 3 & 9 & \\
Methylprednisolone & 0 & 1 & 1 & \\
Infliximab & 0 & 0 & 0 & \\
Myocardial infarction & 0 & 5 & 5 & $<0.001$ \\
Coronary aneurysm at 1-yr follow-up & 0 & & & \\
\hline
\end{tabular}

Values are presented as numbers.

Group 1, those who exhibited no lesions from acute phase coronary aneurysms; group 2, those who manifested lesions from acute phase aneurysms.

Table 2. Initial laboratory findings of patients

\begin{tabular}{|c|c|c|c|c|}
\hline Variable & Group 1 ( $n=362)$ & Group $2(\mathrm{n}=30)$ & Total $(n=392)$ & $P$ value \\
\hline White blood cells $\left(\times 10^{3} / \mu \mathrm{L}\right)$ & $14.4(11.0-17.5)$ & $17.0(11.2-21.2)$ & $14.5(11.0-17.8)$ & 0.885 \\
\hline Hemoglobin (g/dL) & $11.2(10.6-11.8)$ & $10.8(9.8-11.3)$ & $11.2(10.6-11.8)$ & 0.001 \\
\hline Platelets $\left(\times 10^{3} / \mu \mathrm{L}\right)$ & $334.5(274.8-393.0)$ & $365.0(288.5-509.8)$ & 337.0 (275.3-395.8) & 0.015 \\
\hline Neutrophil (\%) & $62.1(50.8-72.7)$ & $68.0(47.5-85.5)$ & $62.1(50.2-73.0)$ & 0.566 \\
\hline Aspartate aminotransferase (IU/L) & $37.0(27.8-74.3)$ & $36.0(26.8-70.5)$ & $37.0(27.0-73.0)$ & 0.311 \\
\hline Alanine aminotransferase (IU/L) & $34.0(15.0-137.5)$ & $34.5(18.0-86.3)$ & $34.0(15.3-136.5)$ & 0.665 \\
\hline Total protein (g/dL) & $6.7(6.3-7.1)$ & $6.8(6.2-7.2)$ & $6.7(6.3-7.1)$ & 0.954 \\
\hline Albumin (g/dL) & $4.1(3.8-4.3)$ & $3.9(3.5-4.1)$ & $4.1(3.8-4.3)$ & 0.004 \\
\hline Total bilirubin (mg/dL) & $0.4(0.3-0.6)$ & $0.3(0.3-0.7)$ & $0.4(0.3-0.6)$ & 0.547 \\
\hline Sodium (mmol/L) & $136.0(135.0-138.0)$ & $136.5(134.8-138.0)$ & $136.0(135.0-138.0)$ & 0.386 \\
\hline C-reactive protein (mg/dL) & $6.6(3.7-10.2)$ & $10.3(6.1-17.6)$ & $6.8(3.8-10.8)$ & 0.005 \\
\hline Harada score (point) ${ }^{*}$ & $4.0(3.0-4.0)$ & $4.0(3.0-5.0)$ & $4.0(3.0-4.0)$ & 0.115 \\
\hline
\end{tabular}

Values are presented as median (interquartile range).

Group 1, those who exhibited no lesions from acute phase coronary aneurysms; group 2, those who manifested lesions from acute phase coronary aneurysms.

*Harada score (point) ${ }^{11}$. 
aneurysm (3 subjects). Results obtained from 1-year follow-up observation revealed complete disappearance of lesions from acute phase coronary aneurysms in 25 subjects while lesions sustained in the other five subjects. These 5 subjects consisted of one case of a small aneurysm, 2 cases of medium aneurysms, and 2 cases of large aneurysms (Fig. 1).

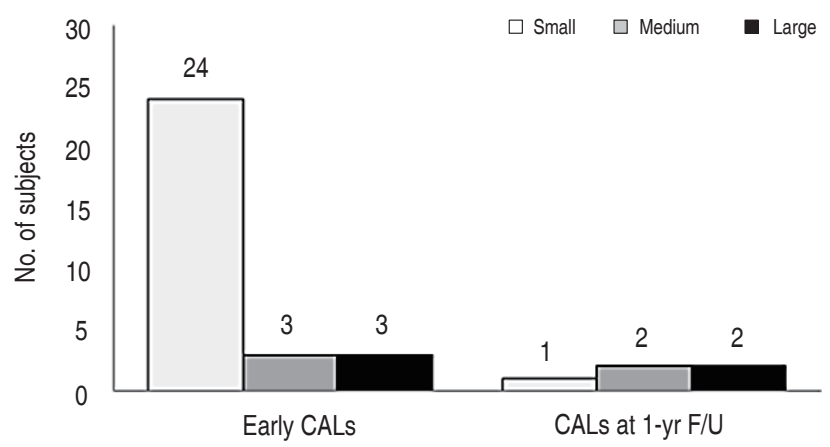

Fig. 1. Changes in coronary arterial lesions (CALS) in early and late followup (F/U). Thirty subjects manifested early CALs, and 24 of them exhibited small aneurysms. The other three subjects showed medium and large aneurysms, respectively. After 1 year, the five remaining subjects comprised 1 case of small, 2 cases of medium, and 2 cases of large aneurysms.
Long duration of the febrile stage $(P<0.001)$, recurrence of the disease $(P=0.002)$, lower hemoglobin count $(P=0.002)$, higher platelet count $(P<0.001)$, lower level of albumin $(P<0.001)$, and higher level of CRP $(P=0.001)$ were found to be significant risk factors for acute phase coronary aneurysms in univariate analysis. In multivariate analysis, long duration of febrile stage $(P=0.018)$ and higher platelet count $(P=0.009)$ remained as significant risk factors (Table 4). Group 2 showed longer duration of the febrile stage (OR, 1.47; 95\% CI, 1.06-2.02; $P=0.018$ ) and higher platelet count (OR, $1.00 ; 95 \%$ CI, $1.00-1.01 ; P=0.009)$.

In univariate analysis, higher level of total WBC $(P=0.028)$ and larger inner diameter of acute phase of coronary artery $(P=0.016)$ were found to be risk factors for coronary aneurysms in patients whose coronary aneurysm was sustained for one year. However, they were found to be insignificant factors in multivariate analysis (Table 5).

Five subjects exhibited coronary aneurysms that were sustained for 1 year. Their characteristics are summarized in Table 6. Of them, subject number 5 manifested comparatively shorter duration of the febrile stage, of five days, that resulted in the occurrence of a coronary aneurysm while 2 subjects among the 5 subjects were diagnosed with complete Kawasaki disease. Four subjects (except

Table 4. Risk factors associated with early coronary aneurysm ( $n=392)$

\begin{tabular}{|c|c|c|c|c|c|c|}
\hline \multirow{2}{*}{ Variable } & \multicolumn{3}{|c|}{ Univariate } & \multicolumn{3}{|c|}{ Multivariate } \\
\hline & Odds ratio & $95 \% \mathrm{Cl}$ & $P$ value & Odds ratio & $95 \% \mathrm{Cl}$ & $P$ value \\
\hline Weight (kg) & 1.10 & $1.02-1.18$ & 0.010 & 1.11 & $0.96-1.28$ & 0.140 \\
\hline Days of fever & 1.62 & $1.33-1.99$ & $<0.001$ & 1.47 & $1.06-2.02$ & 0.018 \\
\hline Recurrence & 4.59 & $1.77-11.8$ & 0.002 & 3.14 & $0.42-23.4$ & 0.264 \\
\hline Refractory KD & 3.163 & $1.25-7.95$ & 0.014 & 2.67 & $0.43-16.56$ & 0.289 \\
\hline Hemoglobin $(\mathrm{g} / \mathrm{dL})$ & 0.45 & $0.28-0.74$ & 0.002 & 0.57 & $0.28-1.15$ & 0.018 \\
\hline Platelets $\left(\times 10^{3} / \mu \mathrm{L}\right)$ & 1.00 & $1.00-1.00$ & $<0.001$ & 1.00 & $1.00-1.01$ & 0.009 \\
\hline Albumin (g/dL) & 0.20 & $0.08-0.48$ & $<0.001$ & 0.31 & $0.04-2.26$ & 0.253 \\
\hline C-reactive protein (mg/dL) & 1.09 & $1.04-1.15$ & 0.001 & 1.00 & $0.90-1.12$ & 0.874 \\
\hline
\end{tabular}

Estimates were adjusted for sex, age, weight, days of fever, complete $\mathrm{KD}$, recurrence, refractory $\mathrm{KD}$, white blood cell count, hemoglobin, platelet, albumin, and $\mathrm{C}$-reactive protein levels in the logistic regression analysis.

$\mathrm{KD}$, Kawasaki disease; $\mathrm{Cl}$, confidence interval.

Table 5. Risk factors associated with late coronary aneurysms in subgroup analysis $(n=30)$

\begin{tabular}{|c|c|c|c|c|c|c|}
\hline \multirow{2}{*}{ Variable } & \multicolumn{3}{|c|}{ Univariate } & \multicolumn{3}{|c|}{ Multivariate } \\
\hline & Odds ratio & $95 \% \mathrm{Cl}$ & $P$ value & Odds ratio & $95 \% \mathrm{Cl}$ & $P$ value \\
\hline Days of fever & 1.37 & $0.94-2.01$ & 0.096 & - & - & - \\
\hline White blood cells $\left(\times 10^{3} / \mu \mathrm{L}\right)$ & 1.17 & $1.01-1.36$ & 0.028 & 1.14 & $0.96-1.34$ & 0.117 \\
\hline Neutrophil (\%) & 1.08 & $0.99-1.17$ & 0.052 & - & - & - \\
\hline Albumin (g/dL) & 0.19 & $0.02-1.83$ & 0.152 & - & - & - \\
\hline C-reactive protein (mg/dL) & 1.13 & $0.98-1.32$ & 0.090 & - & - & - \\
\hline Degree of coronary aneurysm (small vs. medium vs. large) & 46.0 & $2.01-1047.80$ & 0.016 & 4.44 & $0.13-143.58$ & 0.401 \\
\hline
\end{tabular}

Estimates were adjusted for sex, age, weight, days of fever, complete KD, recurrence, refractory KD, white blood cell count, hemoglobin, platelet, albumin, and $\mathrm{C}$-reactive protein levels in the logistic regression analysis.

$\mathrm{KD}$, Kawasaki disease; $\mathrm{Cl}$, confidence interval. 
Table 6. Clinical features of patients with persistent coronary aneurysms

\begin{tabular}{|c|c|c|c|c|c|}
\hline Variable & Patient 1 & Patient 2 & Patient 3 & Patient 4 & Patient 5 \\
\hline Age at diagnosis (mo) & 66 & 15 & 4 & 4 & 45 \\
\hline Days of fever & 8 & 12 & 9 & 6 & 12 \\
\hline Complete Kawasaki disease & Y & $\mathrm{N}$ & Y & $\mathrm{N}$ & $\mathrm{N}$ \\
\hline Platelet $\left(\times 10^{3} / \mu \mathrm{L}\right)$ & 685 & 720 & 461 & 350 & 755 \\
\hline C-reactive protein (mg/dL) & 16.3 & 19.77 & 7.12 & 16.16 & 17.9 \\
\hline Acute treatment & IVIG, ASA & IVIG, ASA, Clopidogrel & IVIG, ASA, Clopidogrel & IVIG, ASA, Clopidogrel & $\begin{array}{l}\text { 2nd IVIG, 3rd mPD, } \\
\text { Infliximab ASA, Clopidogrel }\end{array}$ \\
\hline Echocardiogram at 1-yr follow-up & Large CAL at LAD, RCA & Moderate at LMCA & Moderate at LAD & Small at RCA & $\begin{array}{l}\text { Large at RCA, } \\
\text { moderate at LMCA1 }\end{array}$ \\
\hline Coronary angiography & Y & Y & $\mathrm{N}$ & $\mathrm{N}$ & $\mathrm{N}$ \\
\hline
\end{tabular}

IVIG, intravenous immunoglobulin; ASA, acetylsalicylic acid; mPD, methyl prednisolone; LAD, left anterior descending artery; RCA, right coronary artery; LMCA, left main coronary artery.

subject number 1) were administered with clopidogrel and aspirin in the acute phase while subject number 5 was administered with IVIG (twice), methylprednisolone, and infliximab. These 4 subjects manifested moderate-degree coronary aneurysms (Table 6).

\section{Discussion}

Our data shows that coronary aneurysms were detected in 30 of 392 patients (7.6\%) within 1 month after Kawasaki disease, and medium and large coronary aneurysms were detected in only 6 of 30 patients (20\%). Coronary aneurysms were found to have persisted in five of 30 patients (16.7\%) at 1-year follow-ups. In univariate analysis, significant factors in predicting coronary aneurysm in the early phase, were long duration of fever and high platelet count. Significant factors for persistence of late coronary aneurysms were initial coronary severity and high WBC count.

Since its first report in Japan, Kawasaki disease has shown a higher rate of global incidence since $1967 .^{1,3,8)}$ Since the first report of the disease in Korea in 1973, 194.7 children per 100,000 children aged less than 5 years were diagnosed with Kawasaki disease in 2014 in Korea. ${ }^{8,15,16)}$

Posttreatment convalescence of this disease is comparatively favorable. However, approximately $25 \%$ of cases without treatment can develop coronary aneurysms. ${ }^{2,16,17)}$ Administration of IVIG together with aspirin within 10 days after the onset of this disease can significantly reduce the risk of cardiovascular complications. ${ }^{16,17)}$ Such rarely observed cardiovascular complications can have fatal consequences. Thus, early diagnosis and treatment of this disease together with follow-up observations employing regular echocardiographies are important. ${ }^{2)}$

Tewelde et al. ${ }^{11)}$ have developed a scoring system to evaluate the risk of coronary aneurysms occurring at acute phase or later in children diagnosed with Kawasaki disease. In the present study, Harada scores did not have a significant effect on coronary aneurysms. Other studies have reported risk factors for coronary aneurysms or experiencing no reaction from an initial treatment with IVIG for children diagnosed with Kawasaki disease. ${ }^{10,18)}$ A metaanalysis of 12 previous studies with 2,745 patients in 2016 reported that higher levels of absolute neutrophil count, $N$-terminal pro btype natriuretic peptide, bilirubin, alanine aminotransferase, and CRP and lower levels of albumin and sodium were risk factors for no reaction to initial treatment with IVIG. ${ }^{19)}$ Higher levels of platelet count and CRP and lower levels of hemoglobin and albumin were found to be risk factors for complications of acute phase coronary aneurysms.

Such difference might be due to comparatively early diagnoses of Kawasaki disease recently and a high rate of successful treatment against incomplete Kawasaki disease. ${ }^{7,20)}$ Higher platelet count at the time of diagnosis was found to be a risk factor for the occurrence of coronary aneurysms. This was different from results of other previous studies wherein lower platelet count was presented as a risk factor. Previous results could be ascribable to frequent occurrence of coronary aneurysm in patients with long duration of the febrile stage who showed higher level of platelet counts accordingly.

In the present study, one of subjects with large coronary aneurysms later had complete recovery. There were also cases of small coronary aneurysms that were sustained longer. No critical complications related to coronary aneurysms were found from later observation of outpatients' record. These outpatients are tracked currently through administrations of anticoagulant, regular echocardiographies and special investigations.

Most outpatients with Kawasaki disease are to have complete disappearance of coronary aneurysms. However, the finding of sustained small lesions can support the present study as grounds for granting significance to follow-up observation of characteristics of patients who exhibit sustained coronary aneurysms 1 year after initial treatment.

This study has some limitations. First, this was a retrospective investigation conducted for patients from a single medical insti- 
tution. The number of subjects manifesting coronary aneurysms was insufficient. A definite judgment on the final consequences of coronary aneurysms was unavailable as echocardiographies were conducted 1 year later for these subjects. Thus, considering these limitations, further studies are needed.

Appropriate treatments pertinent to each symptom of coronary aneurysms and regular examinations are desirable to secure favorable convalescence. Additional studies are needed to determine the mechanism involved in the disappearance of coronary aneurysms and the factors involved.

In conclusion, the convalescence from Kawasaki disease of children who took in-patient care from our hospital was favorable overall. Regular echocardiographies are needed due to the risk of the occurrence of coronary aneurysms. Further studies with longterm follow-up observations are needed to examine the presence of complications of Kawasaki disease closely.

\section{Conflicts of interest}

No potential conflict of interest relevant to this article was reported.

\section{References}

1. Lee YH. Kawasaki disease. Yeungnam Univ J Med 2000;17:99-107.

2. Dajani AS, Taubert KA, Gerber MA, Shulman ST, Ferrieri P, Freed M, et al. Diagnosis and therapy of Kawasaki disease in children. Circulation 1993;87:1776-80.

3. Honkanen VE, McCrindle BW, Laxer RM, Feldman BM, Schneider R, Silverman ED. Clinical relevance of the risk factors for coronary artery inflammation in Kawasaki disease. Pediatr Cardiol 2003;24:122-6.

4. Hirata S, Nakamura Y, Yanagawa H. Incidence rate of recurrent Kawasaki disease and related risk factors: from the results of nationwide surveys of Kawasaki disease in Japan. Acta Paediatr 2001;90: 40-4.

5. Lin MT, Sun LC, Wu ET, Wang JK, Lue HC, Wu MH. Acute and late coronary outcomes in 1073 patients with Kawasaki disease with and without intravenous $\gamma$-immunoglobulin therapy. Arch Dis Child
2015;100:542-7.

6. Ha KS, Jang G, Lee J, Lee K, Hong Y, Son C, et al. Incomplete clinical manifestation as a risk factor for coronary artery abnormalities in Kawasaki disease: a meta-analysis. Eur J Pediatr 2013;172:343-9.

7. Kim JJ, Hong YM, Yun SW, Han MK, Lee KY, Song MS, et al. Assessment of risk factors for Korean children with Kawasaki disease. Pediatr Cardiol 2012;33:513-20.

8. Park JW, Chung E, Park K, Jang YT, Park SA. Long term follow-up study of patients with Kawasaki disease. Pediatr Infect Vaccine 2015; 22:164-71.

9. Kim T, Choi W, Woo CW, Choi B, Lee J, Lee K, et al. Predictive risk factors for coronary artery abnormalities in Kawasaki disease. Eur J Pediatr 2007;166:421-5.

10. Newburger JW, Takahashi M, Gerber MA, Gewitz MH, Tani LY, Burns JC, et al. Diagnosis, treatment, and long-term management of Kawasaki disease: a statement for health professionals from the Committee on Rheumatic Fever, Endocarditis, and Kawasaki Disease, Council on Cardiovascular Disease in the Young, American Heart Association. Pediatrics 2004;114:1708-33.

11. Tewelde H, Yoon J, Van Ittersum W, Worley S, Preminger T, Goldfarb J. The Harada score in the US population of children with Kawasaki disease. Hosp Pediatr 2014;4:233-8.

12. Lang B. Recognizing Kawasaki disease. Paediatr Child Health 2001; 6:638-43.

13. Burns JC, Kushner HI, Bastian JF, Shike H, Shimizu C, Matsubara T, et al. Kawasaki disease: a brief history. Pediatrics 2000;106:E27.

14. Jung JW. Long-term outcome of Kawasaki disease complicated by a large coronary aneurysm. Korean Circ J 2017;47:444-5.

15. Kim GB, Han JW, Park YW, Song MS, Hong YM, Cha SH, et al. Epidemiologic features of Kawasaki disease in South Korea: data from nationwide survey, 2009-2011. Pediatr Infect Dis J 2014;33:24-7.

16. Heo MY, Choi SJ, Kim HS, Sohn SJ. Clinical features of atypical Kawasaki disease. J Korean Pediatr Soc 2002;45:376-82.

17. Lee HY, Song MS. Predictive factors of resistance to intravenous immunoglobulin and coronary artery lesions in Kawasaki disease. Korean J Pediatr 2016;59:477-82.

18. Ha KS, Jang GY, Lee J, Lee KC, Son CS. Laboratory markers in incomplete Kawasaki disease according to coronary artery outcome. Korean Circ J 2018;48:287-95.

19. Baek JY, Song MS. Meta-analysis of factors predicting resistance to intravenous immunoglobulin treatment in patients with Kawasaki disease. Korean J Pediatr 2016;59:80-90.

20. Rhim JW, Youn YS, Han JW, Lee SJ, Oh JH, Lee KY. Changes in Kawasaki disease during 2 decades at a single institution in Daejeon, Korea. Pediatr Infect Dis J 2014;33:372-5. 